\title{
SiGe HBT X-Band LNAs for Ultra-Low-Noise Cryogenic Receivers
}

Tushar K. Thrivikraman, Student Member, IEEE, Jiahui Yuan, Student Member, IEEE, Joseph C. Bardin, Student Member, IEEE, Hamdi Mani, Stanley D. Phillips, Student Member, IEEE, Wei-Min Lance Kuo, Member, IEEE, John D. Cressler, Fellow, IEEE, and Sander Weinreb, Life Fellow, IEEE

\begin{abstract}
We report results on the cryogenic operation of two different monolithic X-band silicon-germanium (SiGe) heterojunction bipolar transistor low noise amplifiers (LNAs) implemented in a commercially-available $130 \mathrm{~nm}$ SiGe BiCMOS platform. These SiGe LNAs exhibit a dramatic reduction in noise temperature with cooling, yielding $T_{\text {eff }}$ of less than $21 \mathrm{~K}(0.3 \mathrm{~dB}$ noise figure $)$ across $\mathrm{X}$-band at a $15 \mathrm{~K}$ operating temperature. To the authors' knowledge, these SiGe LNAs exhibit the lowest broadband noise of any Si-based LNA reported to date.
\end{abstract}

Index Terms-Heterojunction bipolar transistor (HBT), low noise amplifiers (LNAs), silicon-germanium (SiGe).

\section{INTRODUCTION}

$\mathbf{S}_{\mathrm{t}}^{\mathrm{n}}$ ILICON-GERMANIUM (SiGe) hetrojunction bipolar transistors (HBTs) have already shown their strengths in conventional high-frequency [radio frequency (RF) to mm-wave] transceiver applications [1]. As we demonstrate here, another exciting application for SiGe HBTs is their use in ultra-low-noise cryogenically-cooled receivers. Recent work has focused on the potential for operating aggressively-scaled SiGe HBTs in the cryogenic environment in order to achieve greater than one-half TeraHertz frequency response [2] and [3]. We extend that work here to focus on cooled amplifier circuits implemented using a commericially-available $130 \mathrm{~nm} \mathrm{SiGe}$ BiCMOS design platform.

One specific cryogenic application for SiGe HBTs are in low noise amplifiers (LNAs) cooled to a few ten's of Kelvins to enhance receiver sensitivity and noise performance for detecting very weak RF signals. Such receivers are routinely used for radio astronomy applications, where at present very costly Gallium-Arsenide (GaAs) and Indium-Phosphide (InP) LNAs are the de facto technology of choice. SiGe HBT LNAs have been recently demonstrated for low frequency cryogenic receivers (1-3 GHz) [4], but there have been no monolithic high frequency $(8-12 \mathrm{GHz}) \mathrm{SiGe}$ HBT cryogenic LNAs reported to date, and this is addressed in the present work for the first time.

Manuscript received January 10, 2008; revised March 17, 2008. This work was supported by Research Institute Fellows Council, Georgia Institute of Technology (Georgia Tech), NASA-ETDP, JPL, and the members of the SiGe Devices and Circuits Group, Georgia Tech.

T. K. Thrivikraman, J. Yuan, S. D. Phillips, W.-M. L. Kuo, and J. D. Cressler are with the School of Electrical and Computer Engineering, Georgia Institute of Technology, Atlanta, GA 30332-0250 USA (e-mail: tthrivi@ece.gatech.edu).

J. C. Bardin, H. Mani, and S. Weinreb are with the Department of Electrical Engineering, California Institute of Technology, Pasadena, CA 91125 USA (e-mail: sweinreb@caltech.edu).

Color versions of one or more of the figures in this letter are available online at http://ieeexplore.ieee.org.

Digital Object Identifier 10.1109/LMWC.2008.925104
SiGe HBTs are a strong candidate for today's RF through mm-wave wireless applications because their bandgap engineering greatly improves both the dc, ac, and noise performance while maintaining very conservative lithographic scaling nodes (e.g., $285 \mathrm{GHz} f_{\max }$ at $130 \mathrm{~nm}$ ). In addition, cooling naturally increases the various performance metrics such as current gain $(\beta)$, cutoff frequency $\left(f_{T}\right)$, and maximum oscillation frequency $\left(f_{\max }\right)$, and also reduces the transistor parasitics such as the base resistance $\left(R_{B}\right)$ and junction capacitances $\left(C_{B C}\right.$ and $C_{B E}$ ). The reduction of $R_{B}$ and $C_{B E}$, in particular, together with the increase of $f_{T}$, should greatly improve the broadband noise performance, which is dominated by base and collector current shot noise and the base thermal noise. Therefore, superior RF performance can indeed be anticipated in SiGe HBTs operating at cryogenic temperatures, making this low-cost Si-based technology a natural candidate for ultra-low-noise cryogenic receivers.

The present work reports measurement results on two different monolithic X-band SiGe LNAs at cryogenic temperatures. We highlight the LNAs performance, including noise and gain across bias and frequency.

\section{LNA Design, Packaging, AND Measurement}

The two SiGe LNAs were designed using the traditional, inductively-degenerated cascode architecture, and implemented in the commercially-avilable, $130 \mathrm{~nm}, 200 / 285 \mathrm{GHz}\left(f_{T} / f_{\max }\right)$, third-generation IBM $8 \mathrm{HP}$ SiGe BiCMOS process. The first LNA (LNA-A) was optimized for achieving less than $1.5 \mathrm{~dB}$ noise figure and $19 \mathrm{~dB}$ of gain at $\mathrm{X}$-band at $300 \mathrm{~K}$ [5]. The second LNA (LNA-B) was optimized for much lower power operation, to achieve $2 \mathrm{~dB}$ noise figure and $10 \mathrm{~dB}$ of gain, while dissipating only $2 \mathrm{~mW}$ of de power [6].

Both SiGe LNAs were measured at room temperature $(300 \mathrm{~K})$ and at deep cryogenic temperature $(15 \mathrm{~K})$. The LNAs were not specifically designed for low temperature operation; however, bias currents were swept to determine the optimum noise performance. The $S$-parameters were measured using an Agilent E8363B PNA in a custom-designed, open-cycle, on-wafer, high-frequency probe station capable of achieving a chuck temperature of $4.5 \mathrm{~K}$ (details of the measurement setup can be found in [3]).

In order to measure the noise performance, the LNAs were packaged to accommodate an available coaxial cryogenic noise measurement system. Each SiGe LNA was mounted in an RF package enclosure with low-loss coaxial K-connectors. A bias-tee was fabricated in package using a single layer capacitor and dc feed resistor. A schematic and photograph for an LNA 

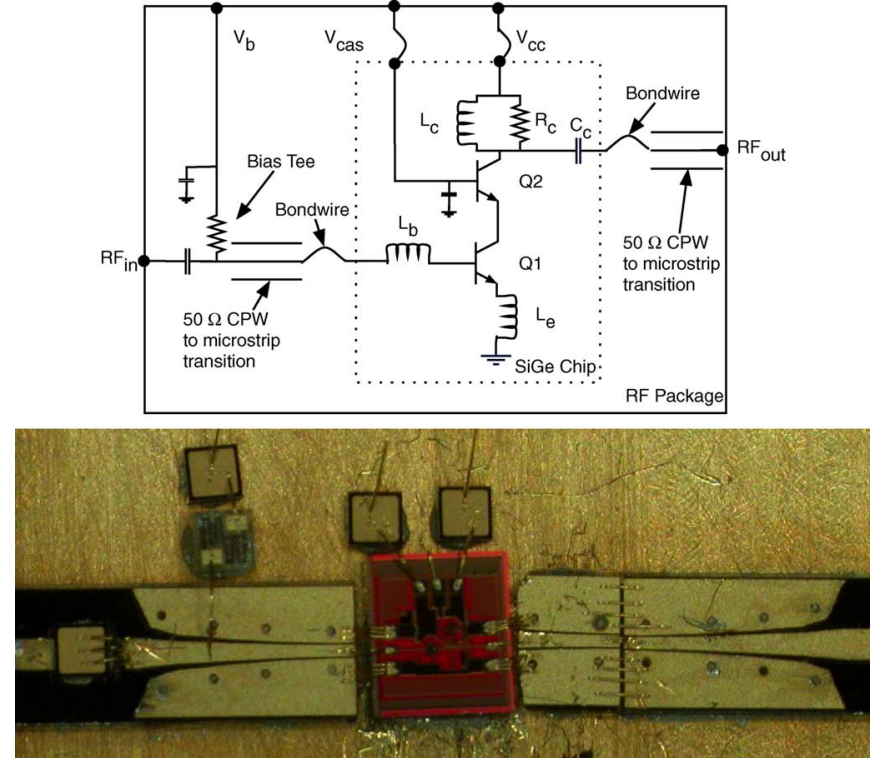

Fig. 1. Schematic and photograph of an X-band SiGe LNA in the package enclosure.

in the package enclosure is shown in Fig. 1. These packages provided only minimal degradation of RF performance compared to on-wafer measurements of the circuits.

The broadband noise measurements were performed using the cold attenuator method and were conducted at the RF and Microwave Group Facility, California Institute of Technology, Pasadena, CA [4]. The cold attenuator method uses a temperature calibrated $20 \mathrm{~dB}$ attenuator at the input of the amplifier to reduce measurement uncertainty, facilitating the accurate measurement of very low noise amplifiers [7]. In order to de-embed the noise temperature of the amplifier, the temperature of the cryogenic attenuator is entered into the Agilent N8975A noise figure analyzer (NFA), which then calculates the corrected noise temperature using the $Y$-factor noise measurement method.

Errors in the NFA and the noise source cause a fixed percentage error in the measured noise, therefore, lowering the termination temperature increases the measurement accuracy. In our case without the cooled attenuator, measuring the $21 \mathrm{~K} \mathrm{am}$ plifier with $1 \mathrm{~K}$ accuracy requires measuring $321 \mathrm{~K}$ ( $300 \mathrm{~K}$ noise source $+21 \mathrm{~K}$ amplifier noise) with $0.3 \%$ error. On the other hand, with the attenuator cooled to $15 \mathrm{~K}$, only $18 \mathrm{~K}$ of noise temperature is added by the termination and $1 \mathrm{~K}$ accuracy only requires $2.5 \%$ error in the measurement.

\section{Measurement Results}

LNA-B was measured using the on-wafer cryogenic probing station to obtain the room temperature $(300 \mathrm{~K})$ and cryogenic (15 K) $S$-parameters. The collector current for the LNA was maintained at a constant $1.3 \mathrm{~mA}$ for both the room and lowtemperature measurements. Fig. 2 shows that $S_{21}$ at $10 \mathrm{GHz}$ increases by approximately $5 \mathrm{~dB}$ with cooling, from $10 \mathrm{~dB}$ at room temperature to $15 \mathrm{~dB}$ at low temperature. Both $S_{11}$ and $S_{22}$ are well matched across the band, showing a return loss better than $10 \mathrm{~dB}$ at $10 \mathrm{GHz}$.

Noise temperature and gain for both LNA-A and LNA-B were measured using the cryogenic noise measurement

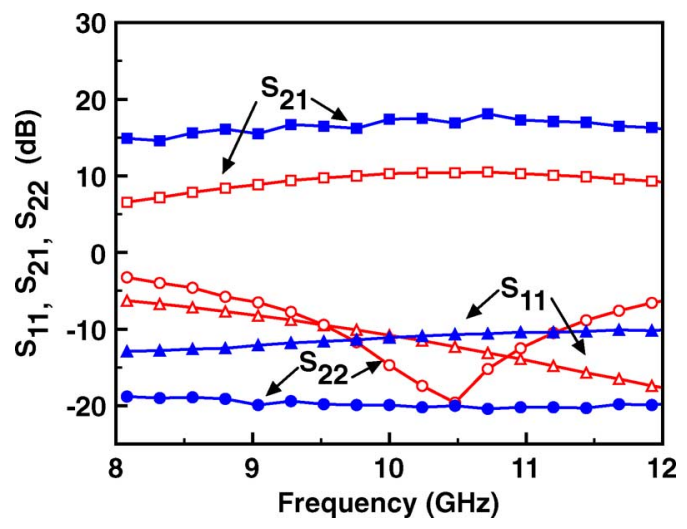

Fig. 2. Comparison of room temperature and low-temperature $S$-parameters for LNA-B, open/closed symbols represent $300 \mathrm{~K} / 15 \mathrm{~K}$, respectively.

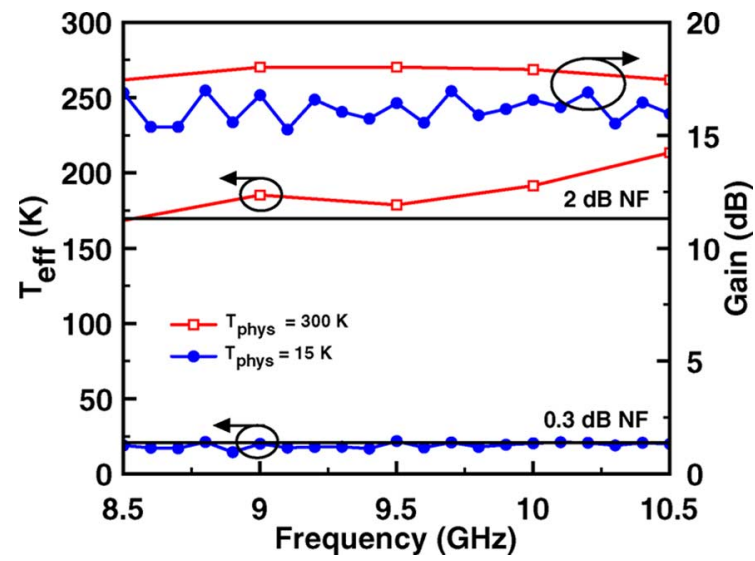

Fig. 3. Effective noise temperature and gain for LNA-A at $15 \mathrm{~K}$ operating temperature, with $I_{C}=1.1 \mathrm{~mA}$.

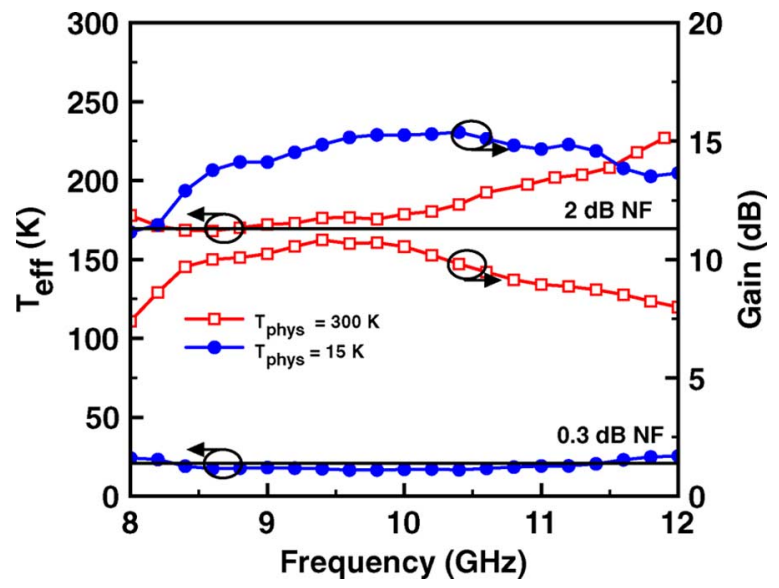

Fig. 4. Effective noise temperature and gain for LNA-B at $15 \mathrm{~K}$ operating temperature, with $I_{C}=1.1 \mathrm{~mA}$.

system. Figs. 3 and 4 show the cryogenic performance of LNA-A/LNA-B, respectively, at $I_{C}=1.1 \mathrm{~mA}$. In both cases, the cryogenic noise performance improves dramatically over the room temperature performance. LNA-A's cryogenic noise temperature decreases to below $21 \mathrm{~K}(0.3 \mathrm{~dB}$ noise figure $)$ at an operating temperature of $15 \mathrm{~K}$, while dissipating only $2.25 \mathrm{~mW}$ of dc power. The decrease in the gain of LNA-A is due to a reduction in $I_{C}$ to accommodate low noise performance at 


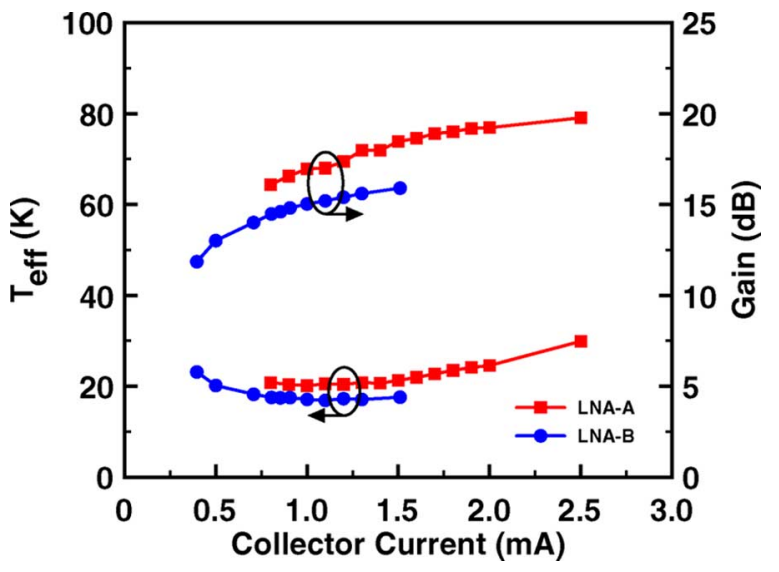

Fig. 5. Noise temperature and gain at $10 \mathrm{GHz}$ for the SiGe LNAs as a function of collector current operating at $15 \mathrm{~K}$ temperature.

cryogenic temperatures. Similar performance improvements for LNA-B are highlighted in Fig. 4, which shows a $150 \mathrm{~K}$ reduction in $T_{\text {eff }}$ and an almost $5 \mathrm{~dB}$ increase in gain across X-band. To the authors' knowledge, these SiGe LNAs exhibit record noise performance for any $\mathrm{Si}$-based LNA operating at $\mathrm{X}$-band. In addition, this noise performance is very competitive with InP HEMT devices which have been shown to have a $T_{\text {eff }}$ of less than $10 \mathrm{~K}$ at X-band [8].

A further examination into the differences between these two LNAs can lead to insight into optimized SiGe LNA design for cryogenic operation. Room temperature simulations indicated that the minimum noise temperature occurs at a collector current density of approximately $1.2 \mathrm{~mA} / \mu \mathrm{m}^{2}$. Fig. 5 plots the effective noise temperature and gain at $10 \mathrm{GHz}$ for both LNA-A and LNA-B over a range of collector currents. This figure indicates that cooling shifts the minimum noise temperature to a bias of $0.25 \mathrm{~mA} / \mu \mathrm{m}^{2}$.

From these data, it can be expected that the low-power optimized LNA-B provides a greater performance improvement with cooling compared to LNA-A because the operating points between the room and low temperature measurements are similar. The bias point of LNA-A was reduced by $5 \mathrm{~mA}$ to provide the optimal noise temperature, resulting in a decrease in gain at cryogenic temperatures. In addition, the LNA-A noise temperature is $5-10 \mathrm{~K}$ higher than for LNA-B due to the differences in optimization of the noise input matching network. These results suggest that low-power optimization for room temperature operation will yield a design better-suited for cryogenic low-noise performance.

\section{CONCLUSION}

We have demonstrated the superior cryogenic capabilities of SiGe HBTs for high-frequency cooled receivers by measuring two different SiGe HBT X-band LNAs: LNA-A, which was optimized for noise performance, and and LNA-B, which was optimized for low power. Both SiGe LNAs were measured to have below $21 \mathrm{~K}$ effective noise temperature and $15 \mathrm{~dB}$ of gain at $15 \mathrm{~K}$ operating temperature. To the authors' knowledge, these results demonstrate the lowest noise performance of any Si-based LNA operating at X-band and highlight the potential for SiGe HBT LNAs for ultra-low-noise cryogenic receiver applications. In addition to the record noise performance reported, we also highlight possible design strategies for optimizing high-frequency SiGe LNAs for cryogenic applications. Due to the scaling of collector currents at minimum noise at cryogenic temperature in SiGe HBTs, design for low-power dissipation results in a more optimized design for low-temperature applications. Future work will focus on exploring this design strategy for wide-band cryogenic receiver front-ends using SiGe HBT technology.

\section{REFERENCES}

[1] J. P. Comeau, M. A. Morton, W.-M. L. Kuo, T. Thrivikraman, J. M. Andrews, C. Grens, J. D. Cressler, J. Papapolymerou, and M. Mitchell, "A monolithic 5-bit SiGe BiCMOS receiver for X-band phased-array radar systems," in Proc. IEEE BCTM Conf., 2007, pp. 172-175.

[2] R. Krithivasan, Y. Lu, J. D. Cressler, J.-S. Rieh, M. H. Khater, D. Ahlgren, and G. Freeman, "Half-terahertz operation of SiGe HBTs," IEEE Electron Device Lett., vol. 27, no. 7, pp. 567-569, Jul. 2006.

[3] J. Yuan, R. Krithivasan, J. D. Cressler, M. H. Khater, D. C. Ahlgren, and A. J. Joseph, "On the frequency limits of SiGe HBTs for terahertz applications," in Proc. IEEE BCTM Conf., 2007, pp. 22-25.

[4] S. Weinreb, J. C. Bardin, and H. Mani, "Design of cryogenic SiGe lownoise amplifiers," IEEE Trans. Microw. Theory Tech., vol. 55, no. 11, pp. 2306-2312, Nov. 2007.

[5] W. M. L. Kuo, Q. Liang, J. D. Cressler, and M. A. Mitchell, "An $\mathrm{X}$-band SiGe LNA with $1.36 \mathrm{~dB}$ mean noise figure for monolithic phased array transmit/receive radar modules," in IEEE RFIC Symp. Dig., 2006, pp. 549-552.

[6] T. K. Thrivikraman, W. M. L. Kuo, J. P. Comeau, A. K. Sutton, J. D. Cressler, P. W. Marshall, and M. A. Mitchell, "A 2 mW, sub-2 $\mathrm{dB}$ noise figure, SiGe low-noise amplifier for X-band high-altitude or space-based radar applications," in IEEE RFIC Symp. Dig., 2007, pp. 629-632.

[7] J. E. Fernandez, "A Noise-Temperature Measurement System Using a Cryogenic Attenuator," Jet Propulsion Laboratory, Pasadena, CA, Tech. Rep. 42-135F, 1998.

[8] R. Grundbacher, R. Lai, M. Barsky, R. Tsai, T. Gaier, S. Weinreb, D. Dawson, J. J. Bautista, J. F. Davis, N. Erickson, T. Block, and A. Oki, "0.1 $\mu \mathrm{m}$ InP HEMT devices and MMICs for cryogenic low noise amplifiers from X-band to W-band," in Proc. Indium Phosphide Related Mater. Conf., 2002, pp. 455-458. 